\title{
A comparative study of the immunity region of lambdoid phages including Shiga-toxin-converting phages: molecular basis for cross immunity.
}

\author{
Kazi Riaz Fattah ${ }^{1}$, Satoshi Mizutani ${ }^{2}$, Farjana Jahan Fattah ${ }^{1}$, \\ Aizo Matsushiro ${ }^{3}$ and Yoshinobu Sugino ${ }^{1 *}$. \\ ${ }^{1}$ Laboratory of Molecular Biology, Kansai Medical University, 18-89 Uyamahigashimachi, Hirakata, \\ Osaka 573-1136, Japan. \\ ${ }^{2}$ Department of Public Health, Kansai Medical University, Moriguchi, Osaka 570-8506, Japan. \\ ${ }^{3}$ The School of Biology-Oriented Science and Technology, Department of Molecular Genetics, \\ Kinki University, Uchita, Wakayama 649-6493, Japan.
}

(Received 29 August 2000, accepted 5 October 2000)

\begin{abstract}
Comparison of eight lambdoid phages, including three Shiga-toxin converting phages, has been carried out with respect to the immunity region, especially the recognition helices of their repressor and CRO proteins on the one hand, and operator sequences on the other. Some as yet unassigned components of the regulatory circuits have been inferred by computer search. The cross immunity phenomenon shown by phages VT2-Sa and lambda is explained on the basis of similarity in their sequences. In addition, the similarity of $933 \mathrm{~W}$ and HK022 in the sequences of their recognition helices of repressor and $\mathrm{CRO}$, on the one hand, and operators, on the other, has led us to predict that they will have identical or similar immunity specificity. This homology has enabled us also to locate the $\mathrm{O}_{\mathrm{L}}$ (and consequently $\mathrm{P}_{\mathrm{L}}$ ) of phage $933 \mathrm{~W}$ that has been thought to be non-existent.
\end{abstract}

\section{INTRODUCTION}

The very early regulation of phage lambda and related phages 434 and P22 has been studied in great detail, and has become a paradigm for regulation of gene expression (Ptashne, 1992). In recent years, the association of Shigalike toxin genes with lambdoid phages has been the cause of renewed interest in these phages and DNA sequences of new lambdoid phages are becoming available (Neely and Friedman, 1998a and 1998b; Plunkett et al., 1999; Miyamoto et al., 1999; Makino et al., 1999).

We have made an exhaustive comparative study of the immunity regions of the three Shiga-toxin converting phages VT2-Sa(VT2-Sakai), H-19B and 933W on the one hand, and lambda, HK022, $\phi 80$, P22 and 434 on the other, and attempted to assign operators and recognition helices of the repressor and CRO proteins of the newly sequenced phages, when they have not been done so already.

The purpose of the present study is to collect these necessary components of the regulatory circuits for the very early transcription of lambdoid phages when they are available in literature, and to supply them by extracting them from the sequence information when they are not

Edited by Hideo Shinagawa

* Corresponding author. E-mail: sugino@makino.kmu.ac.jp already given in literature, so that it would be possible to draw a coherent picture of the common features of these circuits.

It has been shown (Matsushiro, Sato and Miyamoto, to be published) that the toxin-converting phage VT2-Sa shows cross immunity with phage lambda. Thus an $E$. coli K-12 strain non-lysogenic for lambda was first lysogenized with phage VT2-Sa; the resulting lysogen did not form plaques not only of phage VT2-Sa but also of phage lambda. On the other hand, a K-12 lambda lysogen did not form any plaques when plated with phage VT2-Sa. This observation is unique in that no other phage has so for been shown to have cross immunity with phage lambda. (Salmonella phage P22 and coliphage 21 have been shown to have identical immunity by Botstein and Herskowitz, 1974; Poteete et al., 1980). The DNA sequence of the VT2-Sa phage has been published by Miyamoto et al. (1999) who sequenced the DNA isolated from phage particles. Makino et al. (1999) sequenced a closely related sequence in a part of $E$. coli genome DNA containing a prophage called VT2-Sakai. The results of these groups are almost identical, although there are some significant discrepancies between them. Makino et al. concluded that the DNA sequence of the immunity region of VT2-Sakai is not similar to those of lambda, 933W or other known bacteriophages, and consequently that the 
repressor and CRO proteins of VT2-Sakai seem to possess a different recognition specificity from that of any other known phages including lambda (Makino et al., 1999).

\section{MATERIALS AND METHODS}

The DNA sequences were taken from the following

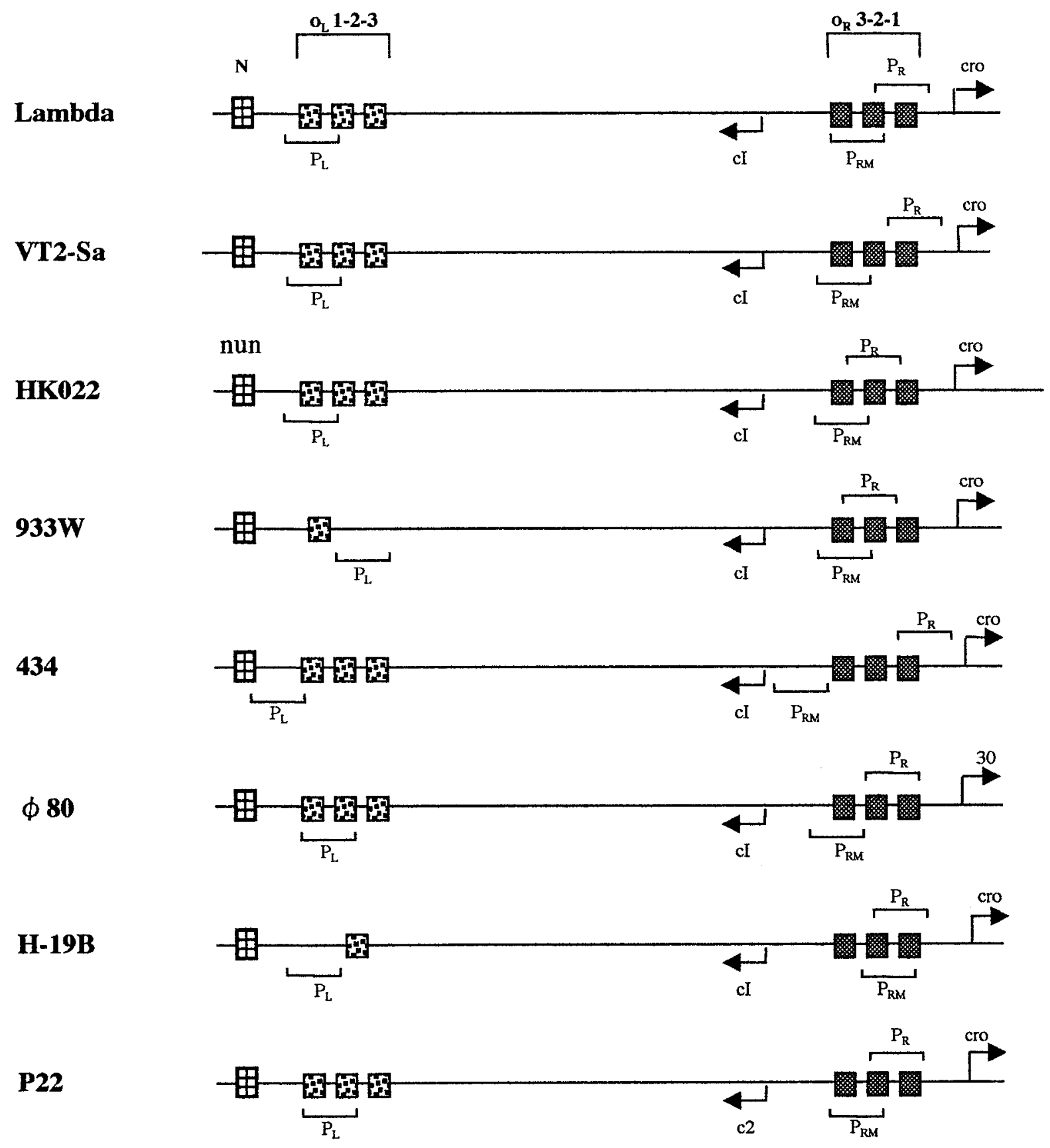

Fig. 1. Organization of the immunity region of lambdoid phages. $P_{R}$ and $P_{L}$ are the rightward and leftward promoters respectively while $\mathrm{P}_{\mathrm{RM}}$ is the promoter for repressor maintenance. The data for lambda was from Ptashne (1992); VT2-Sa (VT2-Sakai) from Makino et al. (1999) and Miyamoto et al. (1999); HK022 from Oberto et al. (1989); 933W from Plunkett et al. (1999); 434 from Branden and Tooze (1991); ф80 from Ogawa et al. (1988); H-19B from Neely and Friedman (1998a) and P22 from Poteete et al. (1980) and Kropinski and VanderByl (unpublished). The elements not provided in literature were found by us by computer search of the known sequence information (See Text). The gene for repressor of P22 in the immunity region is called $c 2$, the $N$ analogue of HK022 nun and the cro analogue of $\phi 80$ is called gene 30 . 
sources: lambda from Daniels et al., 1983 and Ptashne, 1992; VT2-Sa from Miyamoto et al., 1999; VT2-Sakai from Makino et al., 1999; H-19B from Neely and Friedman, 1998a; 933W from Plunkett et al., 1999; HK022 from Oberto et al., 1989; $\phi 80$ from Ogawa et al., 1988; 434 from Branden and Tooze, 1991; and P22 from Pottete et al., 1980 and Kropinski and VanderByl, unpublished.

The sequences of the recognition helices of the phages were obtained from the following sources: lambda and 434 from Branden and Tooze, 1991; HK022, $\phi 80$ and P22 from Oberto et al., 1989.

When operator or promoter sequences or helix-turn-helix motifs were not available in the literature, we searched them in the sequence data by the use of computer softwares. The computer softwares used in this study were DNAMAN, Version 4.1, Lynnon Biosoft, and GENETYXMAC, Version 7.3, Software Development Company Ltd.

\section{RESULTS AND DISCUSSION}

General organization of the immunity region We have made a comparison of the immunity regions of eight lambdoid phages (Fig. 1). The same general pattern is followed in all the phages we studied. The region between the $c r o$ and the $c$ I gene (or the $c 2$ gene in P22) contains the right operator $\left(\mathrm{O}_{\mathrm{R}}\right)$ and the $\mathrm{P}_{\mathrm{R}}$ and $\mathrm{P}_{\mathrm{RM}}$ promoters. The $\mathrm{O}_{\mathrm{R}}$ in all the phages contains three operator sites $\left(\mathrm{O}_{\mathrm{R}} 1\right.$, $\mathrm{O}_{\mathrm{R}} 2$ and $\left.\mathrm{O}_{\mathrm{R}} 3\right)$. The left operators $\left(\mathrm{O}_{\mathrm{L}}\right)$ are present between the $c \mathrm{I}$ and the $N$ gene or their analogues. The $\mathrm{P}_{\mathrm{L}}$ promoter is also present in this region. Although we could identify only one operator site in $\mathrm{O}_{\mathrm{L}}$ for phages $933 \mathrm{~W}$ and $\mathrm{H}-19 \mathrm{~B}, \mathrm{O}_{\mathrm{L}}$ in all other phages have three operator sites. One operator site can be functionally sufficient for $\mathrm{O}_{\mathrm{L}}$, as shown by other systems such as the lac operon.

Thus the immunity region of all these phages are constructed on a similar general plan (Ptashne, 1992), even where the necessary components have been hitherto thought to be missing. The only deviation from the lambda paradigm is that in the $\mathrm{O}_{\mathrm{L}}$ of $933 \mathrm{~W}$ and $\mathrm{H}-19 \mathrm{~B}$, only one operator site could be found whereas in others there are three operator sites in $\mathrm{O}_{\mathrm{L}}$. But here the problem might be why $\mathrm{O}_{\mathrm{L}}$ in these other phages should contain three operator sites, rather than why there is only one operator site in $933 \mathrm{~W}$ or $\mathrm{H}-19 \mathrm{~B}$; since $\mathrm{O}_{\mathrm{L}}$ is not involved in a switch function between two divergently oriented promoters, but seems to be an ordinary operator controlling only one promoter, it does not seem intrinsically necessary to have more than one operator site.

\section{VT2-Sa}

\begin{tabular}{|c|c|c|c|c|c|c|c|c|c|c|c|c|c|c|c|c|c|c|}
\hline & 1 & 2 & 3 & 4 & 5 & 6 & 7 & 8 & 9 & & 1 & 2 & 3 & 4 & 5 & 6 & 7 & 8 \\
\hline \multirow[t]{2}{*}{ OR1 } & A & A & $\mathrm{G}$ & $T$ & A & C & A & $T$ & $A$ & OR1 & $T$ & A & $T$ & C & A & C & C & G \\
\hline & $A$ & $A$ & $A$ & $T$ & $A$ & $C$ & $A$ & $A$ & $T$ & & $T$ & $A$ & $C$ & $C$ & $T$ & C & $\mathrm{T}$ & G \\
\hline \multirow[t]{2}{*}{ OR2 } & A & $\mathrm{T}$ & G & C & A & C & $A$ & $A$ & $T$ & OR2 & $T$ & $A$ & A & C & $A$ & C & C & G \\
\hline & $A$ & A & $A$ & $T$ & A & C & A & $C$ & $A$ & & C & $A$ & A & $C$ & A & C & $\mathbf{G}$ & C \\
\hline \multirow[t]{2}{*}{ OR3 } & $\mathrm{T}$ & A & $T$ & $T$ & A & C & $\mathbf{G}$ & $C$ & $T$ & OR3 & $T$ & $A$ & $T$ & C & A & C & C & G \\
\hline & C & $A$ & $G$ & $T$ & A & C & $A$ & $\mathbf{G}$ & A & & $T$ & $A$ & $T$ & C & C & C & $T$ & $\mathrm{~T}$ \\
\hline \multirow[t]{2}{*}{ OL1 } & $A$ & $A$ & G & $\mathrm{T}$ & A & C & $A$ & $T$ & $A$ & OL1 & $\mathrm{T}$ & $A$ & $T$ & C & A & C & C & G \\
\hline & $T$ & A & $\mathbf{G}$ & $\mathrm{T}$ & A & C & $\mathbf{G}$ & A & $T$ & & $T$ & $A$ & C & C & A & C & $\mathbf{T}$ & G \\
\hline \multirow[t]{2}{*}{ OL2 } & $T$ & A & G & A & $A$ & C & A & $A$ & A & OL2 & $T$ & A & $T$ & C & $\mathrm{T}$ & C & $\mathbf{T}$ & G \\
\hline & $C$ & A & $A$ & $T$ & A & $C$ & A & $A$ & $T$ & & C & A & A & C & A & $C$ & $C$ & G \\
\hline \multirow[t]{2}{*}{ OL3 } & $A$ & A & A & $\mathbf{T}$ & A & C & A & $A$ & A & OL3 & $\mathrm{T}$ & A & $T$ & C & A & C & C & G \\
\hline & $\mathrm{T}$ & $A$ & C & $A$ & $A$ & C & $\mathbf{G}$ & C & $\mathbf{T}$ & & A & A & C & C & A & $T$ & C & $T$ \\
\hline \multirow[t]{3}{*}{ Consensus } & $A_{6}$ & $A_{11}$ & $\mathrm{G}_{6}$ & $T_{9}$ & $A_{12}$ & $C_{12}$ & $\mathrm{~A}_{9}$ & $\mathrm{~T}_{3}$ & $A_{6}$ & Consensus & $T_{9}$ & $A_{12}$ & $\mathrm{~T}_{6}$ & $C_{12}$ & $A_{9}$ & $C_{11}$ & $C_{7}$ & $G_{9}$ \\
\hline & $T_{4}$ & $\mathrm{~T}_{1}$ & $\mathrm{~A}_{4}$ & $A_{2}$ & & & $\mathrm{G}_{3}$ & $A_{5}$ & $T_{6}$ & & $C_{2}$ & & $A_{3}$ & & $T_{2}$ & $T_{1}$ & $T_{4}$ & $T_{2}$ \\
\hline & $\mathrm{C}_{2}$ & & $C_{1}$ & $C_{1}$ & & & & $\mathrm{G}_{3}$ & & & $A_{1}$ & & $A_{3}$ & & $C_{1}$ & & $\mathrm{G}_{1}$ & $\mathrm{C}_{1}$ \\
\hline
\end{tabular}

Fig. 2. Comparison of operator half sites of phages VT2-Sa and lambda. The positions containing identical bases in the consensus sequences are shown in boxes. The data for lambda from Daniels et al. (1983) and Ptashne (1992). The data for VT2-Sa (VT2-Sakai) from Makino et al. (1999). 


\begin{tabular}{|c|c|c|c|c|c|c|c|c|c|c|c|c|c|c|c|c|c|}
\hline & 1 & 2 & 3 & 4 & 5 & 6 & 7 & 8 & & 1 & 2 & 3 & 4 & 5 & 6 & 7 & 8 \\
\hline \multirow[t]{2}{*}{ OR 1} & C & $\mathrm{G}$ & $\mathrm{T}$ & A & C & $T$ & G & A & OR1 & $T$ & G & $A$ & A & C & C & A & $\mathrm{T}$ \\
\hline & $C$ & G & $T$ & A & $C$ & $T$ & $T$ & $T$ & & $\mathrm{~T}$ & G & $A$ & A & C & $T$ & $T$ & A \\
\hline \multirow[t]{2}{*}{ OR2 } & $A$ & A & A & A & C & $\mathrm{T}$ & $T$ & $T$ & OR2 & $T$ & $\mathbf{G}$ & $T$ & A & C & $T$ & A & $T$ \\
\hline & $T$ & $G$ & A & A & C & $T$ & $G$ & A & & G & G & $A$ & $A$ & C & $T$ & G & A \\
\hline \multirow[t]{2}{*}{ OR3 } & $T$ & G & A & $A$ & $C$ & C & $A$ & $T$ & OR3 & $T$ & $G$ & $A$ & A & C & $C$ & A & $\mathrm{T}$ \\
\hline & $C$ & $G$ & $T$ & $A$ & $C$ & $\mathrm{~T}$ & C & $A$ & & $\mathrm{~T}$ & $G$ & $A$ & $A$ & $C$ & $\mathrm{~T}$ & $T$ & A \\
\hline \multirow[t]{6}{*}{ OL1 } & $\mathrm{T}$ & G & $A$ & $A$ & C & C & A & $A$ & OL1 & $T$ & $\mathbf{G}$ & $A$ & $A$ & C & $\mathbf{T}$ & $T$ & $T$ \\
\hline & C & G & $A$ & $A$ & $C$ & $T$ & $A$ & $\mathbf{T}$ & & $T$ & $G$ & $T$ & A & C & $T$ & $T$ & A \\
\hline & & & & & & & & & OL2 & $T$ & $\mathbf{G}$ & $\mathbf{T}$ & $A$ & $C$ & $T$ & A & $\mathrm{T}$ \\
\hline & & & & & & & & & & A & $\mathbf{G}$ & $T$ & $A$ & $C$ & $C$ & $A$ & A \\
\hline & & & & & & & & & OL3 & $T$ & G & A & $\mathbf{G}$ & C & $\mathbf{T}$ & $\mathbf{T}$ & $\mathrm{T}$ \\
\hline & & & & & & & & & & G & $\mathbf{G}$ & $\mathrm{T}$ & $A$ & $T$ & A & $A$ & A \\
\hline \multirow[t]{3}{*}{ Consensus } & $\mathrm{C}_{4}$ & $\mathrm{G}_{7}$ & $A_{5}$ & $A_{8}$ & $\mathrm{C}_{8}$ & $T_{6}$ & $A_{3}$ & $T_{4}$ & Consensus & $T_{9}$ & $G_{12}$ & $A_{7}$ & $A_{11}$ & $C_{11}$ & $T_{8}$ & $A_{6}$ & $\mathrm{~T}_{6}$ \\
\hline & $T_{3}$ & $A_{1}$ & $T_{3}$ & & & $C_{2}$ & $T_{2}$ & $A_{4}$ & & $\mathrm{G}_{2}$ & & $T_{5}$ & $\mathrm{G}_{1}$ & $T_{1}$ & $\mathrm{C}_{3}$ & $T_{5}$ & $A_{6}$ \\
\hline & $A_{1}$ & & & & & & $\begin{array}{l}\mathrm{G}_{2} \\
\mathrm{C}_{1}\end{array}$ & & & $A_{1}$ & & & & & $A_{1}$ & $\mathrm{G}_{1}$ & \\
\hline
\end{tabular}

Fig. 3. Comparison between the operator half-sites of phages HK022 and 933W. Identical bases at corresponding positions in the consensus sequences are boxed. The data for HK022 was from Oberto et al. (1989); for $933 \mathrm{~W}$ from Plunkett et al. (1999) except $\mathrm{O}_{\mathrm{L}}$ which was found by computer search in the present paper. Only one $\mathrm{O}_{\mathrm{L}}$ site could be found for $933 \mathrm{~W}$ by the computer search.
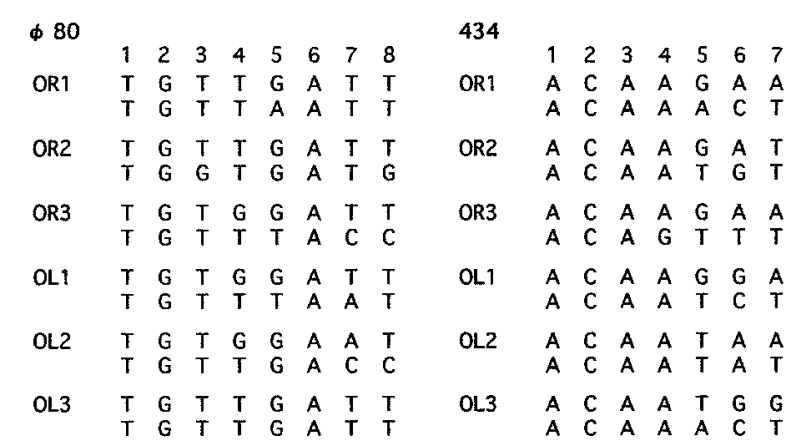

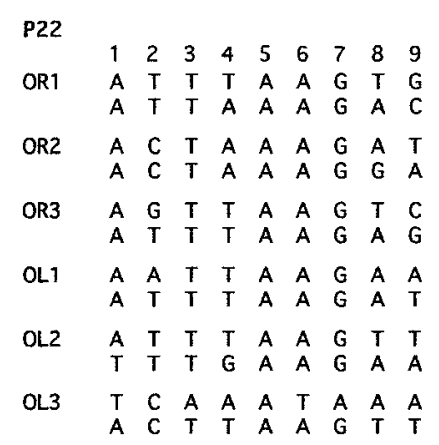

Consensus $T_{12} G_{12} T_{11} T_{9} G_{9} A_{12} T_{8} T_{9}$ Consensus $A_{12} C_{12} A_{12} A_{11} T_{6} \quad A_{5} T_{7}$ Consensus $A_{10} T_{6} T_{11} T_{7} A_{12} A_{11} G_{11} A_{7} T_{4}$

$$
\begin{aligned}
& \begin{array}{lllll}
\mathrm{G}_{1} & \mathrm{G}_{3} & \mathrm{~T}_{2} & \mathrm{~A}_{2} & \mathrm{C}_{2}
\end{array} \\
& \begin{array}{lll}
A_{1} & C_{2} & G_{1}
\end{array} \\
& \begin{array}{lllll}
G_{1} & G_{4} & G_{3} & A_{4}
\end{array} \\
& \begin{array}{llll}
A_{2} & C_{3} & G_{1}
\end{array} \\
& T_{1} \\
& \begin{array}{cccccccc}
T_{2} & C_{4} & A_{1} & A_{4} & T_{1} & A_{1} & T_{4} & A_{4} \\
& A_{1} & G_{1} & & & G_{1} & G_{2} \\
& G_{4} & & & & & C_{2}
\end{array}
\end{aligned}
$$

Fig. 4. Operator half-sites for different lambdoid phages. The data were collected, for $\phi 80$ from Ogawa et al. (1988); 434 from Branden and Tooze (1991); P22 from Pottete et al. (1980) and H-19B from Neely and Friedman (1998a). We located the O $\mathrm{L}_{\mathrm{L}}$ of H-19B by homology search with DNAMAN with the consensus sequence for its $\mathrm{O}_{\mathrm{R}}$ sites as the probe. 
We have been able to point out two pairs of examples where a cross immunity phenomenon is observed or expected from the protein and operator site information. But similar analysis of more lambdoid phages would be necessary to begin to classify the different possible types of immunity specificity.

Operators An operator site has an imperfect dyad symmetry, to which a pair of the recognition helices of both repressor and CRO protein dimers is known to bind (Ptashne, 1992).

The phages we studied can be classified according to the number of base pairs present in the operator sites. 434 has 14 base pairs in its operator site; 933W, HK022 and $\phi 80$ have 15; lambda, VT2-Sa (and VT2-Sakai) and H-19B have 17, while P22 contains 18.

In Fig. 2, 3, and 4 we show the operator sites of lambda and VT2-Sa (and VT2-Sakai), 933W and HK022, and other phages, respectively. We first used the consensus sequence of $933 \mathrm{~W} \mathrm{O}_{R}$ to search for its $\mathrm{O}_{\mathrm{L}}$ in vain, but the $\mathrm{O}_{R}$ and helix-turn-helix motifs of repressor and CRO of $933 \mathrm{~W}$ show remarkable similarity to those of HK022, so we were able to locate an operator site in the $c \mathrm{I}-N$ region of $933 \mathrm{~W}$ by using the operator consensus sequence of phage HK022 as keyword to search the $c \mathrm{I}-N$ region of $933 \mathrm{~W}$ DNA, though $\mathrm{O}_{\mathrm{L}}$ was thought to be non-existent in $933 \mathrm{~W}$ (Fig. 1 and 3 ) (Plunkett et al., 1999). Neely and Friedman mentioned the presence of one $\mathrm{O}_{\mathrm{L}}$ in the regulatory region of early genes of phage $\mathrm{H}-19 \mathrm{~B}$, but its position and sequence was not described in their paper (Neely and Friedman, 1998a). We searched for the sequence and location of $\mathrm{O}_{\mathrm{L}}$ by using the $\mathrm{O}_{\mathrm{R}}$ consensus sequence and could identify one $\mathrm{O}_{\mathrm{L}}$ site (Fig. 1 and 4 ) between the $c \mathrm{I}$ and $N$ gene of $\mathrm{H}-19 \mathrm{~B}$.

Helix-turn-helix motifs The binding of repressor and CRO protein to an operator site is mediated by a helixturn-helix motif, the second helix being responsible for the specificity (Ptashne, 1992; Wharton et al., 1984; Wharton and Ptashne, 1985).

Fig. 5 shows a comparison of the helix-turn-helix motifs

\begin{tabular}{|c|c|c|c|c|c|c|c|c|c|c|c|c|c|c|c|c|c|c|c|c|c|c|}
\hline & \multicolumn{7}{|c|}{ Helix 2} & \multicolumn{5}{|c|}{ Turn } & \multicolumn{10}{|c|}{ Helix 3} \\
\hline & 1 & 2 & 3 & 4 & 5 & 6 & 7 & & 1 & 2 & 3 & 4 & 1 & 2 & 3 & 4 & 5 & $\epsilon$ & & 7 & 8 & 9 \\
\hline Lambda Rep & Q & E & $\mathrm{S}$ & V & A & $D$ & $\mathrm{~K}$ & & $M$ & G & M & $\mathrm{G}$ & $Q$ & $\mathrm{~S}$ & G & V & G & & a & - & $\mathrm{F}$ & $\mathrm{N}$ \\
\hline Lambda Cro & $Q$ & $\mathrm{~T}$ & K & $\mathrm{T}$ & A & $K$ & D & & $L$ & G & V & $Y$ & $Q$ & $\mathrm{~S}$ & A & I & $\mathrm{N}$ & & & 4 & 1 & $\mathrm{H}$ \\
\hline VT2-Sa Rep & Q & $E$ & $k$ & L & A & $E$ & $E$ & & L & G & $M$ & $T$ & $Q$ & G & G & I & G & t & 1 & N & L & $\mathrm{R}$ \\
\hline VT2-Sa Cro & $Q$ & $\mathrm{~T}$ & $T$ & L & A & $K$ & A & & V & G & C & $\mathrm{T}$ & $\mathrm{Q}$ & $\mathrm{G}$ & $\mathrm{A}$ & 1 & G & $⺊$ & 1 & $N$ & $E$ & $S$ \\
\hline HKO22 Rep & $Q$ & A & D & L & A & V & $\mathbf{R}$ & & L & K & V & $T$ & P & K & A & 1 & $\mathrm{~s}$ & & & $N$ & $F$ & $\mathbf{N}$ \\
\hline HK022 Cro & V & $S$ & V & V & A & $D$ & V & & C & G & $L$ & $T$ & $P$ & $K$ & A & I & Y & $\mathrm{k}$ & 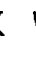 & $N$ & $L$ & $\mathrm{E}$ \\
\hline 933W Rep & $\mathrm{G}$ & M & A & 1 & A & $\mathbf{R}$ & A & & $L$ & S & $L$ & $\mathrm{~s}$ & $\mathrm{~S}$ & K & G & V & $\mathrm{s}$ & $r$ & & N & $F$ & $N$ \\
\hline 933W Cro & & $\mathrm{P}$ & $E$ & V & A & $\mathrm{K}$ & A & & $\mathrm{C}$ & G & V & $S$ & $E$ & $\mathbf{R}$ & $\mathrm{A}$ & V & $Y$ & & & $N$ & $L$ & $K$ \\
\hline H-19B Rep & $L$ & D & $E$ & Q & K & K & I & & A & G & $N$ & $\mathrm{~T}$ & $\mathrm{~N}$ & A & $\mathrm{S}$ & $F$ & A & & ) & K & $L$ & G \\
\hline H-19B Cro & $A$ & $A$ & L & A & $\mathrm{K}$ & $T$ & $\mathrm{~S}$ & & V & G & $Y$ & $\mathbf{L}$ & D & $\mathrm{Q}$ & 1 & A & Y & & & $F$ & $\mathbf{R}$ & $\mathbf{R}$ \\
\hline P22 Rep & $Q$ & A & A & L & G & K & $M$ & & V & G & V & S & $N$ & V & A & 1 & s & ( & 2 & $N$ & E & $\mathbf{R}$ \\
\hline P22 Cro & $Q$ & $\mathbf{R}$ & A & V & A & K & A & & $L$ & G & I & $S$ & D & A & A & V & S & & & $N$ & K & $E$ \\
\hline$\phi 80$ Rep & $Q$ & $\mathbf{R}$ & D & L & A & $\mathbf{E}$ & A & & $L$ & $\mathbf{S}$ & $T$ & $\mathrm{~S}$ & $\mathbf{P}$ & $\mathrm{Q}$ & $\mathrm{T}$ & V & $\Lambda$ & & J & $N$ & 1 & K \\
\hline$\phi 80$ Cro & $Q$ & K & V & L & A & $E$ & K & & V & G & V & $\mathrm{T}$ & $\mathbf{P}$ & $\mathrm{Q}$ & A & 1 & $\Lambda$ & & & $L$ & K & $K$ \\
\hline 434 Rep & $Q$ & A & $E$ & $L$ & A & $Q$ & $K$ & & V & G & $T$ & $T$ & $Q$ & $\mathrm{Q}$ & $\mathrm{S}$ & 1 & 0 & & l & L & $E$ & $N$ \\
\hline 434 Cro & $Q$ & $T$ & E & L & A & $T$ & $\mathrm{~K}$ & & A & G & V & $K$ & Q & $\mathrm{Q}$ & $\mathrm{S}$ & 1 & 5 & & & 1 & $E$ & A \\
\hline
\end{tabular}

Fig. 5. The helix-turn-helix motifs of lambdoid phages. Helices 3 are the recognition helices of the repressor (Rep) and CRO proteins. For details of the source of literature information, or, where they are not available, how we have found them, see text. 
of the lambdoid phages studied in the present work. The motifs for VT2-Sa (and VT2-Sakai) repressor and CRO were found by alignment of their amino acid sequences against the corresponding sequences of lambda, while those of HK022 given in the literature (Oberto et al., 1989) were used to find the helix-turn-helix motifs of repressor and CRO proteins of $933 \mathrm{~W}$. The motif for the repressor protein of $\mathrm{H}-19 \mathrm{~B}$ was assigned from secondary structure prediction while that for its CRO protein was assigned by eye comparison against the corresponding region of HK022 and supported by secondary structure prediction by DNAMAN. Other helix-turn-helix motifs are taken from literature: those for lambda and 434 from Branden and Tooze, 1991, those for HK022, P22 and $\phi 80$ from Oberto et al., 1989.

Promoters The relative positions of the promoters $P_{R}$, $\mathrm{P}_{\mathrm{L}}$, and $\mathrm{P}_{\mathrm{RM}}$ of the eight phages are shown in Fig. 1. The $\mathrm{P}_{\mathrm{L}}$ of phage $933 \mathrm{~W}$, which was not given in the reference, was obtained by computer search by us following the search for $\mathrm{O}_{\mathrm{L}}$.

Oberto et al. (1989) states that they could not detect any transcript corresponding to a $\mathrm{P}_{\mathrm{RM}}$ in their in vitro transcription system for HK022, but this does not mean that there is no $\mathrm{P}_{\mathrm{RM}}$; perhaps some factor, e.g., the repressor, needed for transcription from a $\mathrm{P}_{\mathrm{RM}}$, was missing in the in vitro system. We have been able to find a possible $\mathrm{P}_{\mathrm{RM}}$ sequence for HK022 at the expected site by a computer search.

Comparison between VT2-Sa and lambda. In the assignment of VT2-Sa repressor reading frame in Miyamoto et al. (Miyamoto et al., 1999) there is an extremely good sequence agreement with that of lambda in the C-terminal dimerizing region (Fig. 6A), which abruptly breaks down in the N-terminal DNA-binding domain. This was in part because the DNA sequenced was actually of a $c \mathrm{I}$ mutant that had been inadvertently enriched during the course of repeated vegetative growth. The sequence given by Makino et al. for VT2-Sakai was of a defective prophage integrated in the chromosome of the host $E$. coli, but its $c$ I coding region differs by only one base from that of Miyamoto et al.; thus Miyamoto et al.'s DNA has an amber mutation in the $c \mathrm{I}$ gene (Makino et al., 1999). Alignment of the amino acid sequences of repressor and CRO of lambda and VT2-Sa (or VT2-Sakai) are

\section{A}

Lambda MSTKKKPLTQEQLEDARRLKAIYEKKKNELGLSSESVADKMGMGQSGVGA

VT2-Sa MKWYELARSRMKELGITQEKLAEELGMTQGGIGH

$\begin{array}{ll}\text { Lambda } & \text { LFNGINALNAYNAALLAKIIKVSVEEFSPSIARE IYEMYEAVSMQPSLRS } \\ \text { VT2-Sa } & \text { WLRGSRHPSLSDIGVVFKYLGIDNISENHDGTFSPVGEYSSAPVKKQ... }\end{array}$

Lambda EYEYPVFSHVQAGMFSPELRTFTKGDAERWVSTTKKASDSAFWLEVEGNS

VT2-Sa .YEYPVFSHVQAGMFSPELRTFTKGDAERLVSTTKKASDSAFWLEVEGNS

Lambda MTAPTGSKPSFPDGMLILVDPEQAVEPGDFCIARLGGDEFTFKKLIRDSG

VT2-Sa MTAPTGSKPSFPDGMLILVDPEQAVEPGDFCIARLGGDEFTFKKLIRDSG

Lambda QVFLQPLNPQYPMIPCNESCSVVGKVIASQWPEETFG

VT2-Sa QVFLQPLNPQYPMIPCNESCSVVGKVIAGQWPEETFG

$\mathrm{B}$

Lambda MEQRITLKDYAMRFGQTKTAKDLGVYQSAINKAIHAGRKIFLTINADGSV

VT2-Sa MSNLRKYRESLNISQTTLAKAVGCTQGAIGHWESGRRFPDLKTCRALVA

Lambda YAEEVKPFPSNKKTTA

VT2-Sa CLNKLGAKVSLDDVFPPEHKAA

Fig. 6. The alignment of the repressor and CRO amino acid sequences of VT2-Sa and lambda. The helix-turn-helix motifs are boxed. Identical amino acid residues at corresponding positions are shaded in black while similar residues are shaded in gray. A. repressor; B. CRO. 
shown in Fig. 6A and B. The helix-turn-helix motif of the VT2-Sa repressor was, as noted in a previous section, found by homology search with that of lambda repressor as the keyword. This assignment could be supported by secondary structure prediction using DNAMAN software.

Fig. 2 shows a comparison between the operator consensus sequences of the phages lambda and VT2-Sa (or VT2Sakai). Both phages contain 17 base pairs in their operator sites. Positions 2, 5 and 6 are occupied by identical bases A, A and C, respectively. The recognition helices of repressor and CRO proteins of phages lambda and VT2-Sa also show high similarities (Fig. 5 and 6). The recognition helices of repressor and CRO proteins of lambda bind to operator sites using amino acids on one face of the helix, i.e., amino acids at positions 1,2 , and 6 , and in some cases amino acid at position 5 is also used, while the amino acid at position 4 is used for contact with the other helix in the motifs (Ptashne, 1992).
The recognition helix of repressor of phage VT2-Sa has amino acids at positions 1 , and $5(\mathrm{Q}$, and G respectively) identical to and the amino acid at position 2 ( $\mathrm{S}$ to $\mathrm{G}$ ) similar to the lambda repressor. The recognition helices of the CRO proteins of these phages have identical amino acids at position 1 (Q) and similar amino acids at position 2 (S and G). Thus position 1 (and perhaps position 5) of the recognition helices of repressor or CRO of one of these phages can interact with the operator sequences of the other. It is thus possible for the recognition helix of one phage to recognize and bind to the operator sites of the other and vice versa; an explanation for the cross immunity shown by these phages.

We also note however that there are three common amino acid residues between the recognition helix of repressor and CRO of VT2-Sa which are not shared by those of lambda repressor or lambda CRO, and two of these (at position 2 and 5) are in the face of the helix that is sup-

\begin{abstract}
A
933W MVONEKVRKEFAORLAOACKEAGLDEHGR GMAIARALLLSSKGVSKWFNA

HK022 MVO.QKERETFSORLALACDKAGLPLHGRQADLAVRLKVTPKAISKWFNG

933W ESLPRQEKMNALAKFLNVDVVWLQHGTSLNGANDEDTLSFVGKLKKGLVR HK022 ESIPRKDKMESLASVLGTTAAYL. HGYADDDGITVNHLSRSNDYYRVDVL

933W VVGEAILGVDG ..........AIEMTEERDGWLKIYSDDPDAFGLRVKGD

HK022 DVQASAGPGTMVSNËFIEKIRAIEYTTEQARILFNGRPQESVKVITVRGD
\end{abstract}

\begin{abstract}
933W SMWPRIKSGEYVLIEPNTKVFPGDEVFVRTVEGHNMIKVLGYDRDGEYQF HK022 SMEGTINPGDEIFVDVSITCEDGDGIYVFVYGKTMHVKRLQMQKNRLAVI
\end{abstract}

\author{
933W TSINQDHRPITLPYHQVAKVEYVAGILKQSRHLDDIEAREWLKISS \\ HK022 SDNAAYDRWYIEEGEEEQLHILAKVLIRQSIDYKRFG........
}

B

933W
HK022 MNENLDEPTKGVGI PEVAKACGVSERAVYKWLKNGFLPKTEFFGKTKKA
MNEVEVIKSVRVSVVADVCGLTPKAIYKWLERGSLPRTEFTGETEYA

\title{
933W SKTEEISGGKYOASEMLETSKKNLLAA
}

HK022 DKIAKASGGKYSAAQIRRIGKQQFVM.

Fig. 7. The alignment of the amino acid sequences of repressor and CRO of 933W and HK022. The helix-turn-helix motifs are boxed. A black shade is used when the amino acids at the same position are identical while a gray shade indicates that the two amino acid residues are similar. A, repressor; B. CRO. 


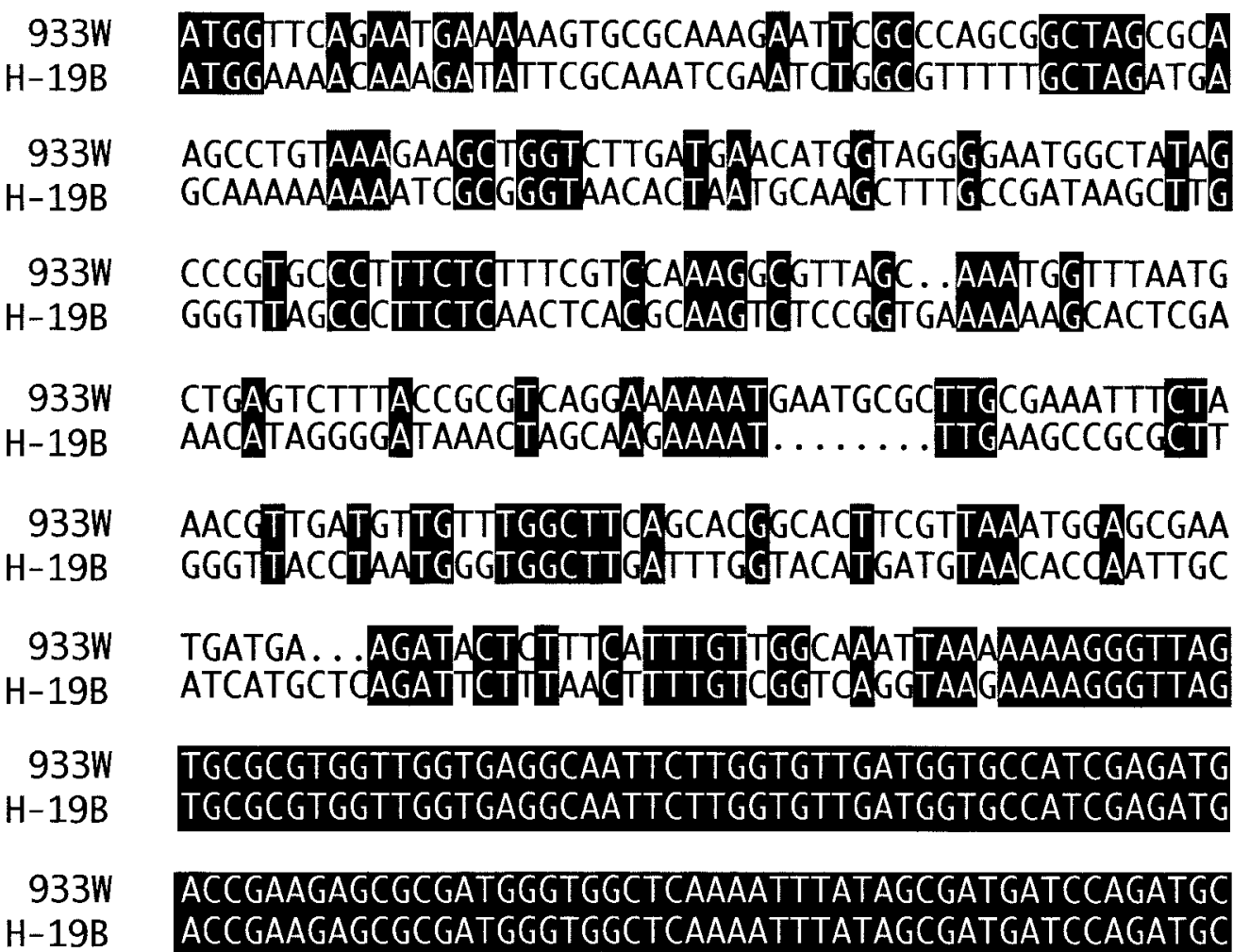

933W CTTTGGTCTTCGTGTGAAAGGAGACAGCATGTGGCCCAGAATAAAATCAG H-19B CTTTGGTCTTCGTGTGAAAGGAGACAGCATGTGGCCCAGAATAAAATCAG

933W GAGAATATGTACTCATTGAGCCTAACACCAAAGTATTCCCGGGTGATGAG H-19B GAGAATATGTACTCATTGAGCCTAACACCAAAGTATTCCCGGGTGATGAG

933W GTGTTTGTCAGAACCGTTGAAGGACACAACATGATTAAGGTTCTTGGC.T H-19B GTGTTTGTCAGAACTGTTGAAGGACACAACATGATCAAAGTTCTTGGCAT

933W ATGACAGAGATGGAGAATACCAATTTACAAGCATTAACCAGGATCACAGG H-19B ATGACAGAGACGGAGAATACCAATTTACAAGCATCAACCAGGACCACAGG

933W CCTATAACGTTGCCTTATCATCAAGTAGCAAAGGTGGAGTATGTAGCTGG $\mathrm{H}-19 \mathrm{~B}$ CCAATAACCTTGCCTTATCATCAAGTAGCAAAGGTGGAGTATGTGGCTGG

933W TATTCTGAAGCAATCTCGCCATCTGGATGACATCGAGGCAAGGGAGTGGC

H-19B TATTCTGAAGCAATCTCGCCATCTGGATGACATCGAGGCAAGGGAGTGGC

933W TGAAAAGTTCGTGA

H-19B TGAAAAGTTCGTGA

Fig. 8. The alignment of nucleotide sequences of 933W and H-19B repressor genes. For H-19B, the off frame sequence after the stop codon corresponding to that of $933 \mathrm{~W}$ and its stop codon is also shown. The stop codons are indicated by stars. The arrow indicates the base, the hypothetical deletion of which will lead to further alignment of the C-terminal domains of the repressors. 
posed to interact with the operator site bases (Fig. 5).

Comparison between 933W and HK022 The operator consensus sequences of HK022 and 933W show striking similarity (Fig. 3). Seven out of eight positions are occupied by identical bases (positions 2-7 of both phages contain $\mathrm{G}, \mathrm{A}, \mathrm{A}, \mathrm{C}, \mathrm{T}, \mathrm{A}$ at respective positions, and the central base pair T/A at position 8 is also common to both phages). The alignment of the repressor and CRO of 933W and HK022 are shown in Fig. 7 A and B. Plunkett et al. (1999) have noted that the $\mathrm{N}$-terminal domain of 933W repressor was very similar to that of HK022, whereas the C-terminal domain was similar to that of $\mathrm{H}-19 \mathrm{~B}$. Indeed, we have found that the recognition helices of the repressor (and CRO) proteins of $933 \mathrm{~W}$ and HK022 show high similarities (Fig. 5). In the repressor proteins, positions $2,5,6,7,8$, and 9 from the $\mathrm{N}$-terminal position of helices 3 contain identical amino acids (K, S, K, $\mathrm{W}, \mathrm{F}$ and $\mathrm{N}$, respectively) while position 4 has similar amino acids ( $\mathrm{V}$ and $\mathrm{I})$. For the CRO proteins, amino acids at positions $3,5,6,7$ and 8 are identical (A, Y, K, W, and L, respectively) while positions 2 and 4 ( $\mathrm{R}$ and $\mathrm{K}, \mathrm{V}$ and I respectively) are similar.

Plunkett et al. could not make any assignment for the $\mathrm{O}_{\mathrm{L}}\left(\right.$ and $\mathrm{P}_{\mathrm{L}}$ ) for $933 \mathrm{~W}$ phage and suggested that an entirely novel mechanism different form the lambda paradigm might be operating here (Plunkett et al., 1999). But similarity of the $\mathrm{O}_{R}$ consensus sequences of phages $933 \mathrm{~W}$ and HK022 (Fig. 3) has prompted us to use the HK022 operator consensus sequence as the probe for a successful search for $933 \mathrm{~W} \mathrm{O}_{\mathrm{L}}$. Thus it is at least too early to suggest that an entirely different regulation mechanism from that of lambda might be operative in $933 \mathrm{~W}$ for its very early leftward transcription (Plunkett et al., 1999).

Since it is highly likely for the recognition helix of repressor of CRO of 933W to recognize and bind to the operator of HK022 and vice-versa, we predict that these two phages will manifest cross immunity specificity.

H-19B and other phages $\mathrm{H}-19 \mathrm{~B}$ has the same number of bases (17) in its operator sites as lambda and VT2-Sa, but its operator sites show little homology with the operator sites of these two phages. H-19B is generally homologous with 933W (Plunkett et al., 1999; Neely and Friedman, 1998a), but it is known to have a different immune specificity from that of 933W (O'Brien et al., 1984). In particular, H-19B repressor has a C-terminal domain (the dimer forming domain), identical with part of 933W repressor, but its $\mathrm{N}$-terminal domain (the DNA binding domain) is entirely different from that of 933W, which is homologous to that of HK022 (Plunkett et al., 1999) (Fig. 7A). The nucleotide sequence of the repressor gene for H-19B has an even higher homology than the amino-acid sequence to that of $933 \mathrm{~W}$; the H-19 repressor lacks the C-terminal 54 amino-acid part of the $933 \mathrm{~W}$ repressor; but if one $\mathrm{A} / \mathrm{T}$ pair were deleted (at coordinate 4737 in the sequence with Accession Number AF034975 in the DNA database), this entire 54 amino acid sequence identical with that of $933 \mathrm{~W}$ counterpart would become attached to its C-terminus (Fig. 8).

The published sequence of the N-terminal domain of $\mathrm{H}-19 \mathrm{~B}$ repressor does not give any appropriate helix-turnhelix motif which can have any homology with its CRO counterpart (Fig. 7B). We tentatively show the helixturn-helix motifs that are possible for CRO and repressor based on secondary structure prediction, but they are mutually not homologous, in sharp contrast with the other phages (Fig. 5). However, it is still possible that $\mathrm{CRO}$ and repressor of H-19B with their dissimilar recognition helices can both interact with the operator site.

$\phi 80$ repressor has a C-terminal domain that has some homology with corresponding sequences of $\mathrm{H}-19 \mathrm{~B}$ and 933W, but has little homology in the N-terminal domain.

In the comparison of the recognition helices and operator sequences, similarities are found only in lambda-VT2Sa and 933W-HK022 pairs. Other pairs show no such high degree of similarity as to suggest cross immunity.

\section{REFERENCES}

Botstein, D. and Herskowitz, I. (1974) Properties of hybrids between Salmonella phage P22 and coliphage $\lambda$. Nature 251, 584-589.

Branden, C. and Tooze, J. (1991) Introduction to Protein Structure. Garland Publishing, Inc. New York and London.

Daniels, D. L., Schroeder, J. L., Szybalski, W., Sanger, F., Coulson, A. R., Hong, G. F., Hill, D. F., Peterson, G. B. and Blattner, F. R. (1983) Complete Annotated Lambda Sequence In: Lambda II (eds. Hendrix, R. W., Roberts, J. W., Stahl, F. W and Weisberg, R. A.), Cold Spring Harbor Laboratory.

Makino, K., Yokoyama, K., Kubota, Y., Yutsudo, C. H., Kimura, S., Kurokawa, K., Ishii, K., Hattori, M., Tatsuno, I., Abe, H., Iida, T., Yamamoto, K., Onishi, M., Hayashi, T., Yasunaga, T., Honda, T., Sasakawa, C., and Shinagawa, H. (1999) Complete nucleotide sequence of the prophage VT2-Sakai carrying the verotoxin 2 genes of the enterohemorrhagic $E s$ cherichia coli 0157:H7 derived from the Sakai outbreak. Genes Genet. Sys. 74, 227-239.

Miyamoto, H., Nakai, W., Yajima, N., Fujibayashi, A., Higuchi, T., Sato, K. and Matsushiro, A. (1999) Sequence Analysis of Stx2-converting Phage VT2-Sa Shows a Great Divergence in Early Regulation and Replication Regions. DNA Res. 6, 235240.

Neely, M. N. and Friedman, D. I. (1998a) Arrangement and functional identification of genes in the regulatory region of lambdoid phage H-19B, a carrier of a Shiga-like toxin. Gene 223, 105-113.

Neely, M. N. and Friedman, D. I. (1998b) Functional and genetic analysis of regulatory regions of coliphage H-19B: location of shiga-like toxin and lysis genes suggest a role for phage functions in toxin release. Mol. Microbiol. 28 (6), 1255-1267.

Oberto, J., Weisberg, R. A. and Gottesman, M. E. (1989) Structure and Function of the nun Gene and the Immunity Region of the Lambdoid Phage HK022. J. Mol. Biol. 207, 675-693.

O'Brien, A. D., Newland, J. W., Miller, S. F., Holmes, R. K., Smith, H. W. and Formal, S. B. (1984) Shiga-like toxin-converting 
phages from Escherichia coli strains that cause hemorrhagic colitis or infantile diarrhea. Science 226, 694-696.

Ogawa, T., Masukata, H. and Tomizawa, J. (1988) Transcriptional Regulation of Early Functions of Bacteriophage $\phi 80$. J. Mol. Biol. 202, 551-563.

Plunkett, G. III, Rose, D. J., Durfee, T. J. and Blattner, F. R. (1999) Sequence of Shiga Toxin 2 phage 933W from Escherichia coli 0157:H7: Shiga Toxin as a Phage Late-Gene Product. J. Bacteriol. 181(6), 1767-1778.

Pottete, A. R., Ptashne, M., Ballivet, M. and Eisen, H. (1980) Op- erator Sequences of Bacteriophages P22 and 21. J. Mol. Biol. 137, 81-91.

Ptashne, M. (1992) A Genetic Switch. 2nd Edition. Cell Press, Cambridge, MA.

Wharton, R. P., Brown, E. L. and Ptashne, M. (1984) Substituting an $\alpha$-helix switches the sequence-specific DNA interactions of a repressor. Cell 38, 361-369.

Wharton, R. P. and Ptashne, M. (1985) Changing the binding specificity of a repressor by redesigning an $\alpha$-helix. Nature 316, 601-605. 